\title{
Correction to: Interactive Segmentation via Deep Learning and B-Spline Explicit Active Surfaces
}

Helena Williams, João Pedrosa, Laura Cattani, Susanne Housmans,

Tom Vercauteren, Jan Deprest, and Jan D’hooge

\author{
Correction to: \\ Chapter "Interactive Segmentation via Deep Learning \\ and B-Spline Explicit Active Surfaces" in: M. de Bruijne et al. \\ (Eds.): Medical Image Computing and Computer Assisted \\ Intervention - MICCAI 2021, LNCS 12901, \\ https://doi.org/10.1007/978-3-030-87193-2_30
}

The original version of this chapter was revised. The figure 2 with missing information was corrected. 